\title{
Calibration of Compact Electron Spectrometer for the FIREX-I Project in Gekko XII
}

\author{
Tetsuo OZAKI, Mayuko KOGA ${ }^{1)}$, Hiroyuki SHIRAGA ${ }^{1)}$, Ryuko KATO ${ }^{2)}$, Shigeru KASHIWAGI ${ }^{2}$, \\ Goro ISOYAMA $^{2}$, Hitoshi SAKAGAMI and FIREX GROUP ${ }^{1)}$ \\ National Institute for Fusion Science, 322-6 Oroshi-cho, Toki 509-5292, Japan \\ ${ }^{1)}$ Institute of Laser Engineering, Osaka University, 2-6 Yamadaoka, Suita 565-0871, Japan \\ ${ }^{2)}$ Institute of Scientific and Industrial Research, Osaka University, 8-1 Mihogaoka, Ibaraki 567-0047, Japan
}

(Received 19 December 2009 / Accepted 23 March 2010)

\begin{abstract}
We have developed a compact electron spectrometer (ESM) to investigate ignition mechanism in the Fast Ignition Realization Experiment project. Hot electrons, produced by the irradiation of a gold target by using a compressed chirped pulse laser, are used for auxiliary heating of the imploded core. An imaging plate is used as the electron detector for medical purposes. However, the relationship between the beam intensity and the detector signal is not clear. The ESM should be calibrated because it is highly compact and has a complex magnetic field for bending caused by installation in a limited space. We have performed the calibration by using an L-band LINAC at the Institute of Scientific and Industrial Research, Osaka University, in order to obtain an accurate electron spectrum. The calibration used a single electron pulse at two different energies, $9.5 \mathrm{MeV}$ and $27.1 \mathrm{MeV}$, with $0.1-10 \mathrm{pC}$. The energy spreads are $0.2 \mathrm{MeV}$ at $9.5 \mathrm{MeV}$ and $0.3 \mathrm{MeV}$ at $27.1 \mathrm{MeV}$. The analyzer has been tested to measure energetic electrons from plain and integrated targets irradiated by the Laser or Fast Ignition Experiment (maximum energy of $10 \mathrm{~kJ}$ ) up to $800 \mathrm{~J}$.
\end{abstract}

(c) 2010 The Japan Society of Plasma Science and Nuclear Fusion Research

Keywords: ESM, calibration, LINAC, electron, FIREX, IP, Gekko XII, LFEX, energy spectrum

DOI: $10.1585 /$ pfr.5.S2098

\section{Introduction}

In laser fusion, the implosion and central ignition of a DT micro balloon have been the most popular methods for realizing inertial confinement fusion [1]. However, they entail a large inconsistency because efficient implosion requires a low temperature, but central ignition at the final stage of the implosion requires produce a high temperature. Therefore, a high laser energy (over $1 \mathrm{MJ}$ ) is necessary. At the Institute of Laser Engineering, Osaka University, the fast ignition concept has been proposed [2], in which core heating is imploded using a highly energetic electron beam produced by the pulse-compressed laser.

High-energy electrons are generated by a strong electric field on the basis of interaction between a high intensity laser and preplasma produced by the laser prepulse [3]. The electron beam energy spectrum strongly depends on the preplasma scale length and the main pulse intensity [4]. To obtain electron energy of less than several $\mathrm{MeV}$, which is suitable for fast ignition, the preplasma scale length should be suppressed sufficiently by selecting appropriate target materials, prepulse control, and target configuration.

In the Fast Ignition Realization Experiment (FIREX) project [5], electrons generated from a gold guiding cone irradiated using a heating laser are used to heat the im-

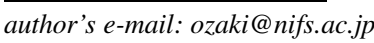

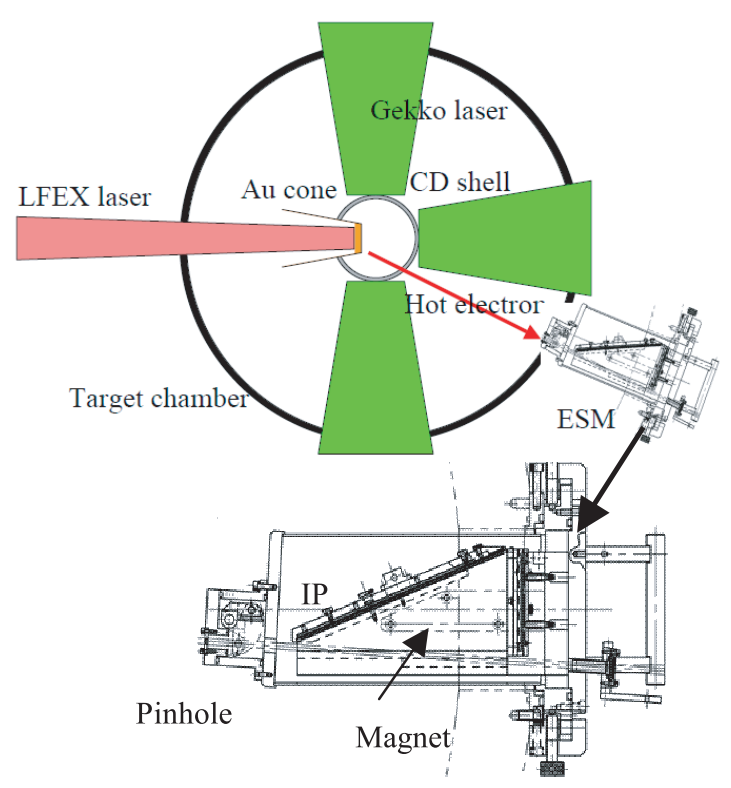

Fig. 1 ESM and experimental arrangement.

ploded core and initiate the fusion burn, as shown in Fig. 1. The highly energetic electron measurement is one of the most important issues in researching the ignition mechanism. Therefore we have developed a compact electron spectrometer (ESM). 
The electron spectra obtained by the ESM do not directly reflect the electron profile in the imploded core, since the electrons are decelerated by an electric field because of space charge produced by an intense electron emission [5]. Therefore the low energy part (below $0.5 \mathrm{MeV}$ ) cannot be observed because of the electrostatic potential. However, the electron spectrum including the low-energy part, can be obtained by extrapolating of the high-energy tail of the electron spectrum if we assume a Maxwellian distribution. Although the results should be compared with those of X-ray spectroscopy [6] or Cherenkov radiation [7], the ESM is still an important tool for investigating the heating mechanism in the core region.

The ESM is highly compact so that it can be installed in a limited space. An imaging plate (IP) intended for medical purposes, is used as the electron detector. Because the relationship between the beam intensity and the detector signal is not clear, the ESM should be calibrated to obtain accurate electron spectra.

\section{Design}

The number and size of the viewing ports are limited in the Gekko XII (GXII) [8] target chamber. All equipment should fit within the virtual conical area created by the center target and a 6-inch port flange. A triangular magnet is selected or its compactness and a wide energy range. The magnet is hidden by the target chamber, thus, saving space. The electron beam enters the analyzer obliquely to obtain a wide observable energy range.

A neodymium alloy is used as the permanent magnet [9]. A magnetic circuit is selected to minimize the magnetic field leakage. However, a small amount of magnetic field leakage remains near the top of the triangle. The twodimensional magnetic field is measured at distances of every $5 \mathrm{~mm}$. The typical magnetic field strength and gap are $0.7 \mathrm{~T}$ and $8 \mathrm{~mm}$, respectively. The beam orbit calculated using the observed magnetic field (Fig. 4).

Inexpensive, commercial IPs (Durr Dental Co.) [10] are used as the beam detectors. This has the advantages of producing no electrical noise and providing a wide dynamic range intensity. Two IP holders are installed for measurements in the high- and low-energy regions to extend the observable energy range. A lead X-ray shield 3$10 \mathrm{~cm}$ thick covers the IP holders to avoid strong X-ray radiation from the target. The IPs are also shielded from light by a shutter in the holder. The shutter is opened just before installation. The holders with the light shield are brought to the IP reader (Vista Scan, Durr Dental Co., $12.5 \mu \mathrm{m} / \mathrm{step}$, $40 \mathrm{LP} / \mathrm{mm}$ ) after the electron irradiation. The data have 16 bit resolution. The IP is irradiated within an $8 \mathrm{~mm}$ width. Therefore, the real signal is obtained by excluding the signals within the $8 \mathrm{~mm}$ width from the background in other areas of the IP.

Alignment is performed from the viewing port behind the beam line and the two-dimensional adjusting mechanism. The analyzer is separated by a small gate valve so that the holders can be removed without breaking the vacuum the GXII target chamber. After the IPs are installed, the analyzer chamber is evacuated using its evacuation system to a vacuum level same as that of the target chamber. Figure 1 shows a schematic diagram of the ESM. The total length and diameter of the main part are $30 \mathrm{~cm}$ and $15 \mathrm{~cm}$, respectively.

\section{Calibration}

The purpose of the calibration is to compare the beam orbit with the calculation and calibrate the beam intensity. We have calibrated of the analyzer by using an L-band LINAC [11] at the Institute of Scientific and Industrial Research, Osaka University. The L-band LINAC can generate strong, ultra-short electron pulses of a maximum of $91 \mathrm{nC}$ and $20 \mathrm{ps}$. Calibration is been performed using single electron pulse at two different energies, $11 \mathrm{MeV}$ and $27 \mathrm{MeV}$. The main energies in the calibration were 9.5 and $27.1 \mathrm{MeV}$, respectively and the charge were 0.1 and $10 \mathrm{pC}$, respectively. The charge is measured by a Faraday cup a $5 \mathrm{~cm}$-thick aluminum rod and an electro meter (Keithley 6514). This penetration width is sufficient to capture energetic electrons. The electron beam from the LINAC passes through air. A vacuum chamber is prepared to minimize beam scattering by air. The beam size is $5 \mathrm{~mm}$ at the exit of the beam line and $10 \mathrm{~mm}$ at a distance of $13 \mathrm{~cm}$ from the exit in air. The energy spreads are $0.2 \mathrm{MeV}$ at $9.5 \mathrm{MeV}$ and $0.3 \mathrm{MeV}$ at $27.1 \mathrm{MeV}$. Figure 2 shows the schematic diagram of the calibration system.

Figure 3 shows the beam patterns on the IPs for

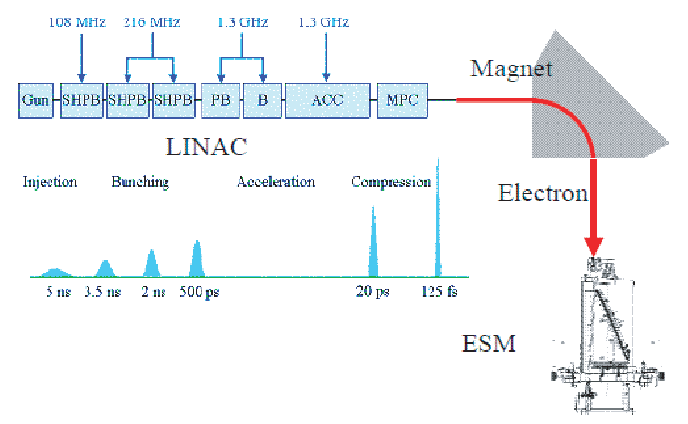

Fig. 2 Schematic diagram of ESM.calibration system.

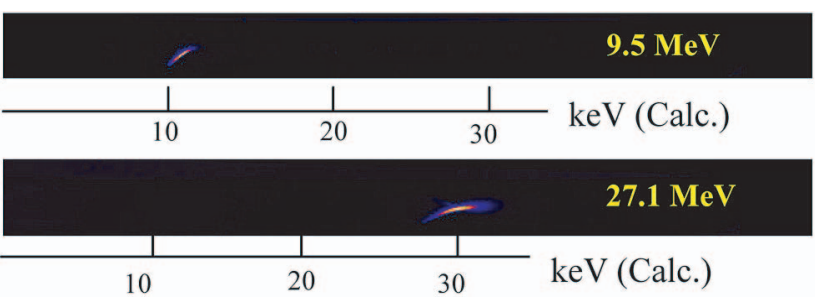

Fig. 3 Electron beam tracks on IPs. 


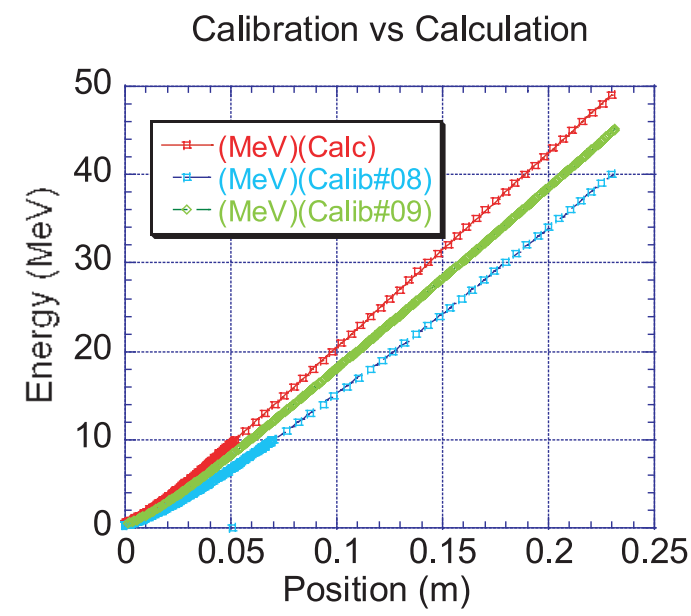

Fig. 4 Electron energy calibration. Solid line with rectangle: calculation, diamond: latest calibration, doted line with rectangle: previous calibration.

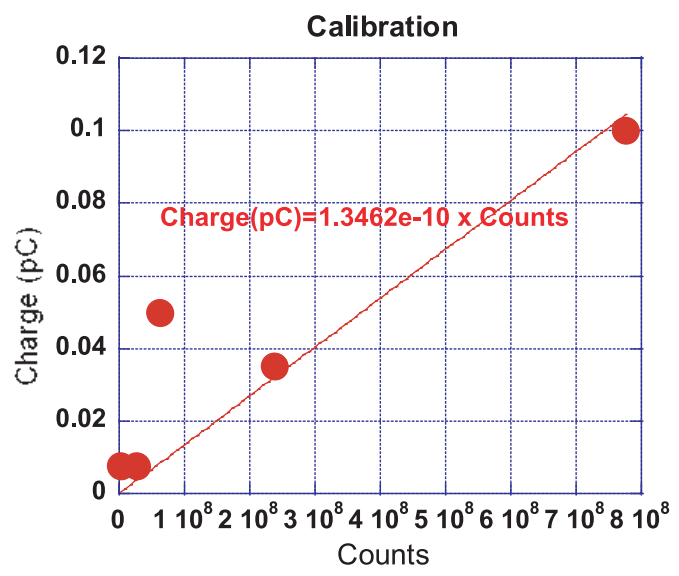

Fig. 5 Electron flux calibration.

9.5 MeV and 27.1 MeV. A part of the beam hits the analyzer wall because of beam expansion. The beam intensity, which is required for our purpose, is almost at the lower limit of the LINAC. The spot position in the calibration is slightly different from the calculated value. The main reason may be the magnetic field leakage near the top of the triangular magnet, as shown in Fig. 1. We obtained the calibration curve from those results, as shown in Fig. 4. In this fitting, a parabola-like magnetic field strength modification is assumed.

The intensity is also calibrated by comparing the incident electrons and the counts on the IP. The background is measured prior to irradiation, and the real signal is obtained by excluding the background from the irradiated signal. Figure 5 shows the result of the intensity calibration.

\section{Target Irradiated by the LFEX Laser}

The analyzer is tested to measure energetic electrons from a plain aluminum target $10 \mathrm{~mm}$ thick, irradiated us-
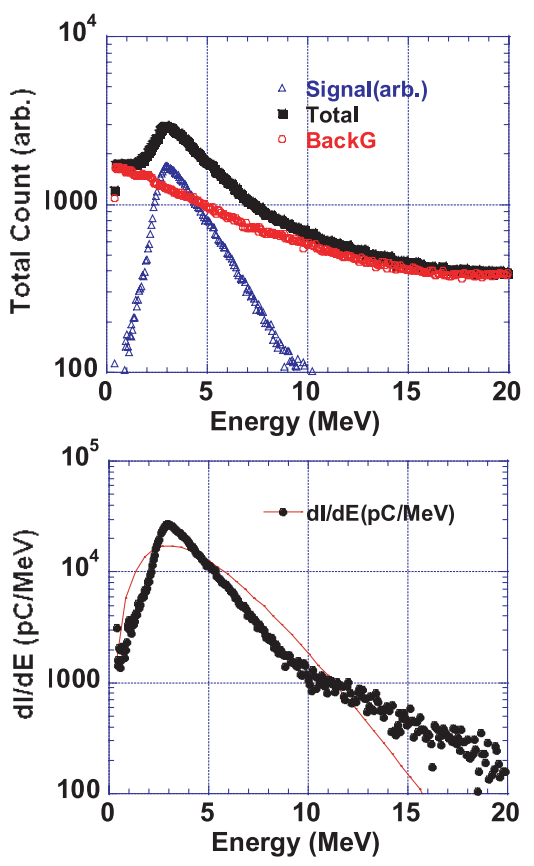

Fig. 6 Typical spectra in the test shot. (a) Closed rectangles: total flux; open circles: background; open triangles: electron flux. (b) Closed circles: energy spectrum; solid line: fitting.

ing the Laser for Fast Ignition Experiment (LFEX) (maximum energy $10 \mathrm{~kJ}$, wavelength $1.05 \mathrm{~mm}$, four beamlets) [12] with an energy of $120 \mathrm{~J}$ and a pulse duration of $4 \mathrm{ps}$. The analyzer is installed on the GXII target chamber at an angle of $20.9^{\circ}$ to the laser injection direction on the back of the target. This shot has was made by compressing one beamlet of the LFEX laser. The analyzer is aligned of the analyzer is performed by viewing a dummy target illuminated by a He-Ne laser. A maximum electron energy of $3 \mathrm{MeV}$ could be observed when the LFEX laser was collimated up to $75 \mathrm{~mm} \times 110 \mathrm{~mm}$ and had an laser intensity of $3.5 \times 10^{17} \mathrm{~W} / \mathrm{cm}^{2}$. Figure 6 shows the energy spectrum of the electron beam. The energy loss of electron in the aluminum foil is written as [13]

$$
\begin{aligned}
& -\frac{\mathrm{d} E}{\mathrm{~d} x}=0.306 \rho \frac{Z}{A} \beta^{2} B, \\
& B=\ln \left(\frac{2 m_{\mathrm{e}} c^{2} \beta^{2}}{I\left(1-\beta^{2}\right)}\right)-\beta^{2},
\end{aligned}
$$

where $\rho, m_{\mathrm{e}}, c, Z, E, A, I$ and $\beta$ are the target density, the electron mass, the light velocity, the target ionization number, the electron energy, the target mass number, the ionization energy and the beam velocity divided by $c$, respectively. The energy loss can be negligible in this energy target although it is extremely important in the imploded target. The ESM detects only the escaped electrons with energies above $0.5 \mathrm{MeV}$, and the number of observed electrons is strongly limited by the high electrostatic potential formed by the electrons.

The IPs are also irradiated by X-rays resulting from the collision of energetic electrons with the target and the 


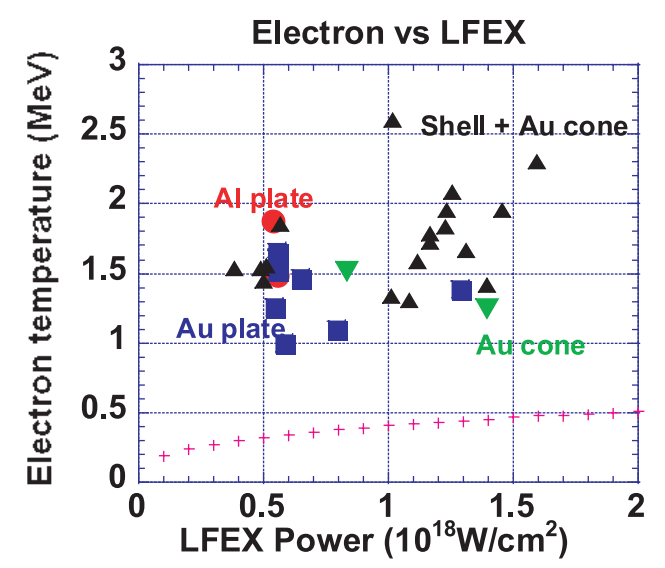

Fig. 7 LFEX power dependence of the hot electron temperature. Plus sign shows the semi-empirical estimation for a petawatt laser. Large discrepancy between data and scaling may result due to the difference in the prepulses.

target chamber. The real electron signal can be obtained by eliminating the X-ray contribution from the signal. Figure 6 shows the spectrum of high-energy electrons from an aluminum foil irradiated by the LFEX laser. The spectrum is obtained from the energy calibration (Fig. 4) and the intensity calibration (Fig. 5). Figure 6(b) shows two different plots, which are derived from the energy calibration factor and orbit calculation using the measured magnetic field. Solid lines indicate the Maxwellian fitting as follows [14]:

$$
\frac{\mathrm{d} N}{\mathrm{~d} E}=\left(\frac{N_{0}}{T}\right)\left(\frac{E}{T}\right)^{2} \exp \left(-\frac{E}{T}\right)
$$

where $N_{0}$, and $T$ are the beam density and effective electron temperature, respectively. Here the relativistic effect is considered because the electron velocity is close to the light velocity. The effective electron temperatures are $0.55 \mathrm{MeV}$ and $0.80 \mathrm{MeV}$, respectively. The observation of energetic electrons indicates that the pulse compression system of the LFEX laser is successfully completed.

We obtained the spectra in Fig. 7 for a different shot. In this shot, the LFEX laser with an energy of $100 \mathrm{~J}$ and a duration of 4 ps was collimated to two spots of $24.5 \mathrm{~mm}$ $\times 44.1 \mathrm{~mm}$ and $24.5 \mathrm{~mm} \times 29.4 \mathrm{~mm}$. The total power was $1.4 \times 10^{18} \mathrm{~W} / \mathrm{cm}^{2}$. The relationship between the effective electron temperature and the laser beam intensity is known as scaling [15], which is semi-empirically derived from the peta-watt laser experiment in GXII [16] as follows:

$$
T_{\mathrm{e}}(M e V)=0.4 \times\left[\left(\frac{I_{\mathrm{L}}\left(W / \mathrm{cm}^{2}\right)}{10^{18}}\right) \lambda(\mu m)^{2}\right]^{1 / 3},
$$

where $T_{\mathrm{e}}$ and $I_{\mathrm{L}}$ are the effective electron temperature and the laser beam intensity, respectively. Figure 7 shows the comparison between the experimental temperature and the empirical temperature. The effective temperature strongly depends on the plasma formed by the prepulse of the LFEX laser. A higher effective temperature in the LFEX laser irradiation than in the peta-watt laser irradiation suggests that a larger prepulse still remains in the LFEX laser. A detailed discussion of the difference temperature will be given elsewhere.

\section{Summary}

The measurement of highly energetic electrons is one of the most important issues fusion ignition mechanism research. We have developed a compact electron spectrometer for be installation in a narrow space. The ESM was calibrated using the L-band LINAC at the Institute of Scientific and Industrial Research, Osaka University. The analyzer is tested to measure energetic electrons from aluminum and gold plain and cone target, and a CD shell with gold cone targets irradiated by the LFEX laser.

A fast ignition integrated experiment by using the imploded core of the GXII and additional heating by the LFEX laser has been started. The ESM results show that the prepulse of the LFEX laser determines the heating quality under present experimental conditions.

[1] H. Azechi et al., Osaka. Laser Part. Beams 9, 193 (1991).

[2] R. Kodama et al., Nature 412, 789 (2001).

[3] M. Key et al., Phys. Plasmas 5, 1966 (1998).

[4] T. Tajima and J. M. Dawson, Phys. Rev. Lett. 43, 267 (1979).

[5] H. Azechi, Plasma Phys. Control. Fusion 48, B267 (2006).

[6] Private communication.

[7] J. Zheng et al., Phys. Plasmas 12, 093105 (2005).

[8] C. Yamanaka et al., proc. 10th Int Conf. London (1984) vol.3, IAEA Vienna (1985).

[9] Private communication.

[10] http://www.duerr.de/

[11] http://www.sanken.osaka-u.ac.jp/labs/rl/facility.html

[12] N. Miyanaga et al., in Proc. of IFSA'03, ThF1.1 (2003).

[13] Private communication.

[14] T. Phillips et al., Rev. Sci. Instrum. 70, 1213 (1999).

[15] Private communication.

[16] Y. Kato et al., Plasma Phys. Control. Fusion 39, 145 (1997). 